Ciencia y tecnología

Science and Technology 



\title{
Sistema de locomoción para discapacitados, automatizado con un procesador myRIO acoplado en una silla de ruedas
}

\author{
Fabricio Paredes Larroca*, Juan Carlos Goñi Delión* \\ Universidad de Lima \\ Recibido: 3 de mayo del 2018 / Aprobado: 4 de julio del 2018 \\ doi: 10.26439/ing.ind2018.n036.2456
}

\begin{abstract}
RESUMEN: La investigación aplicada estudia la posibilidad de desarrollar un sistema de locomoción automatizado para personas con discapacidad. Este sistema permitirá autonomía de desplazamiento a la persona con discapacidad, lo cual mejorará su calidad de vida y le permitirá incluirse con mayor facilidad en la sociedad.
\end{abstract}

Palabras clave: personas con discapacidad / robótica / control automático / silla de ruedas / dispositivos de ayuda para personas con discapacidad / LabVIEW / FPGA / myRIO

\section{Locomotion system for people with disabilities automated with a myRIO processor coupled to a wheelchair}

\begin{abstract}
This applied research studies the possibility of developing an automated locomotion system for people with disabilities. This system will enable said people to have freedom of movement, which will improve their quality of life, and will allow them to be included in society in an easier way.

Keywords: people with disabilities / robotics / automatic control / wheelchairs / self-help devices for people with disabilities / LabVIEW / FPGA / myRIO
\end{abstract}

* Correos electrónicos: fparedes@ulima.edu.pe, jgoni@ulima.edu.pe 


\section{INTRODUCCIÓN'}

La población que presenta en el Perú algún tipo de discapacidad es de un millón seiscientas mil personas aproximadamente (INEI, 2012). De este grupo, el $59 \%$ tiene discapacidad motora o problemas en brazos y piernas, lo que le inhabilita parcial o completamente el caminar.

Asimismo, de este millón seiscientas mil personas, el $51 \%$ tiene problemas visuales o no pueden ver. Y, clasificándolos según su nivel socioeconómico, el $23 \%$ del grupo de discapacitados es de condición pobre o muy pobre. Solo un $20 \%$ de los discapacitados tiene un trabajo, y en casi todos los casos se trata de un trabajo independiente (INEI, 2014). Toda esta información indica las necesidades de ayuda que tienen los peruanos con discapacidad. Ellos necesitan poder ser más independientes que los demás.

\section{PROPUESTA DE SISTEMA DE LOCOMOCIÓN PARA DISCAPACITADOS}

Las personas discapacitadas no tienen libertad para desplazarse de forma segura, y se plantea diseñar un sistema de locomoción que les dé autonomía de movimiento. Para esto se ha planteado la elaboración de un mecanismo mecánico, un sistema eléctrico y un sistema electrónico de control. Todos estos sistemas serán modelados y probados con el uso de software de modelamiento 3D, modelamiento asistido por computadoras (CAD) y simulación de control.

Para cumplir con los objetivos planteados, se ha previsto cumplir con las siguientes características: el peso de una persona promedio de 80 kilogramos, un sistema mecánico de montaje adaptable a sillas de ruedas convencionales, mandos de control de fácil uso, con motores eléctricos de 9 amperios como consumo máximo para 24 voltios; que acceda a rampas de hasta 10 grados de inclinación, con baterías recargables de corriente continua.

\subsection{Análisis del sistema mecánico}

Se ha diseñado el mecanismo de un sistema de placas metálicas adheridas a una silla de ruedas convencional. Estas placas permiten fijar con

1 Este trabajo es auspiciado por el Instituto de Investigación Científica IDIC, de la Universidad de Lima, que busca diseñar e implementar proyectos de gran impacto tecnológico y de ayuda social. 
seguridad un motor eléctrico a cada lado y usar a cambio una rueda de 10 pulgadas de diámetro (con una brida de acoplamiento). Es decir, se deben retirar las ruedas originales de medida promedio de 24 pulgadas para adaptar en un nuevo punto las ruedas pequeñas.

$\mathrm{El}$ acoplamiento entre las placas y los motores cuenta con tres pernos de sujeción para garantizar la seguridad y la estabilidad del conjunto.

El diseño del sistema mecánico se ha basado en la herramienta de dibujo "Diseño Asistido por Computadora" SolidWorks, de la cual se ha establecido la geometría de las placas al ubicar los puntos de apoyo, el equilibrio del peso de la persona, los motores y las baterías. Considerar el centro de gravedad del mecanismo permite el desplazamiento seguro de la silla en superficies horizontales e inclinadas. Asimismo, se han diseñado las plataformas y los soportes para las baterías y los sistemas de control.

La figura 1 muestra la placa de uno de los lados, y en total son dos placas por cada rueda posterior, las cuales serán acopladas a una silla de ruedas convencional que por lo general cuentan con dos ruedas posteriores.

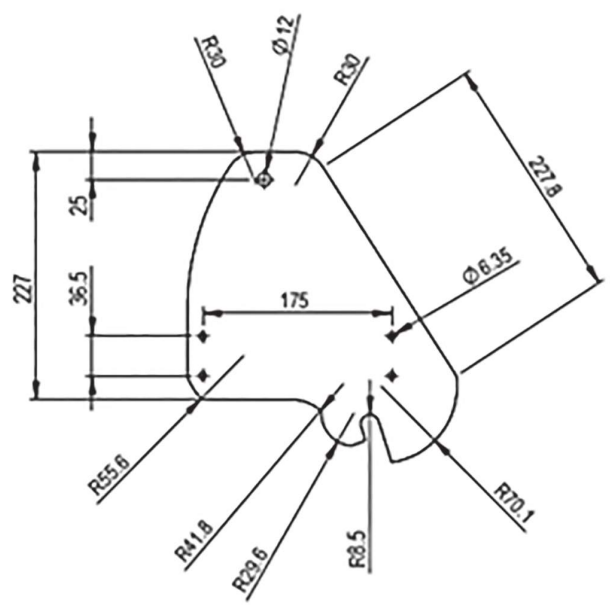

Figura 1. Placa fija de montaje Elaboración propia 
El modelo de locomoción mecánico, además, consta de un sistema adicional de ruedas configurado en estrella que funciona como acoplamiento mecánico capaz de adaptarse a una silla de ruedas estándar para discapacitados, con el objeto de poder subir escaleras con ayuda de dos personas.

Este mecanismo mecánico ha sido concebido, diseñado y fabricado en las instalaciones del laboratorio de Máquinas e Instrumentos de la Universidad de Lima. Para este objetivo, se han utilizado dos conjuntos de ruedas dispuestas en estrella de tres unidades cada uno, unas placas en estrella que sirven para la unión de cada conjunto de ruedas dispuestas en estrella, con bulones de acople para cada una de las seis ruedas y un eje principal extenso para el ensamble a los extremos de los dos conjuntos de ruedas dispuestas en estrella.

La figura 2 muestra el detalle de la placa estrella que funcionará como estructura principal del sistema de acoplamiento de ruedas dispuestas en triángulo.

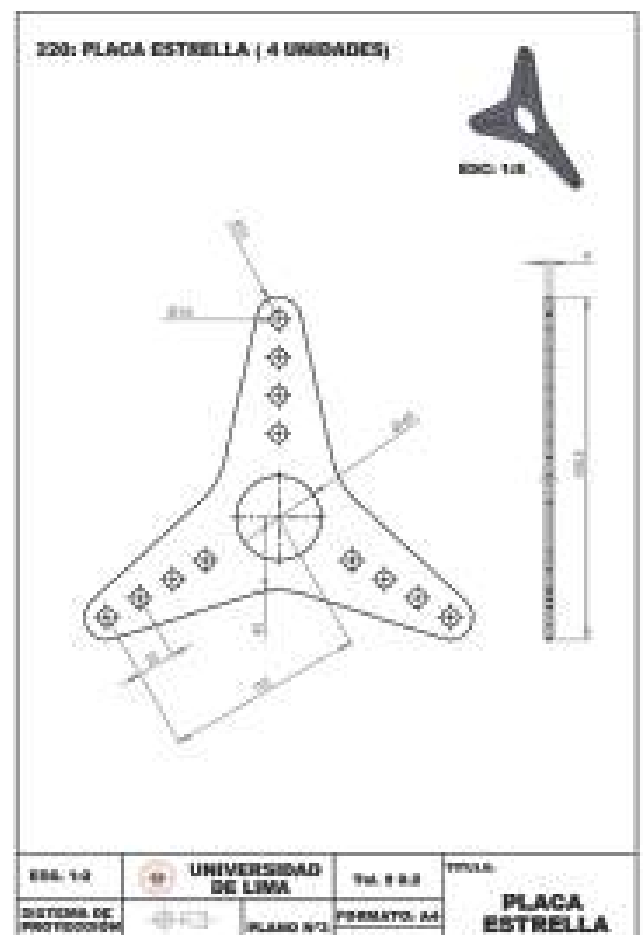

Figura 2. Placa en estrella Elaboración propia 


\subsection{Prototipado y construcción}

El diseño del sistema mecánico de locomoción se ha elaborado con la herramienta de dibujo CAD "Diseño Asistido por Computadora" en SolidWorks hasta obtener el prototipo final. La figura 3 muestra un modelo del conjunto de ruedas dispuesto en estrella.

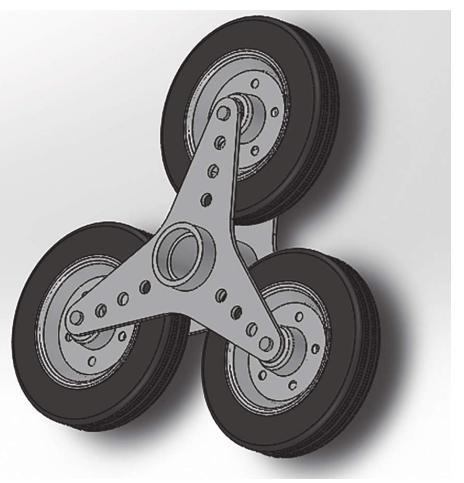

Figura 3. Modelo del sistema de ruedas dispuestas en triángulo Elaboración propia

Se hizo uso de herramientas CAD y CAM (fabricación asistida por computadoras) para el diseño y la fabricación de las piezas que integran el sistema de acople. Las técnicas de manufactura utilizadas fueron: soldadura TIG (del inglés tungsten inert gas), fresado y torneado CNC (torno de control numérico) e impresión 3D.

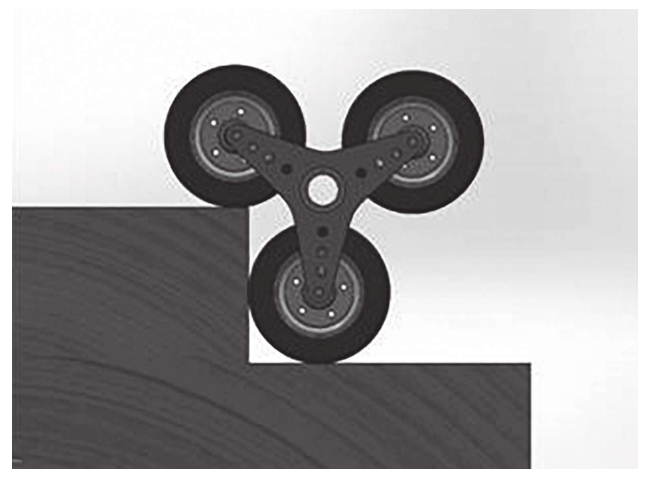

Figura 4. Simulación del acople tipo estrella Elaboración propia 


\subsection{Ensamble y acoplamiento}

Mediante pruebas realizadas con el sistema acoplado a un cargador, se comprobó la efectividad del acople en la superación de obstáculos, y se procedió a realizar el ensamblaje en la silla de ruedas, el cual puede ser retirado e insertado con accionamiento manual. Posteriormente, se realizaron pruebas de campo del prototipo del sistema mecánico en las instalaciones de la Universidad de Lima.

Se acopla el sistema de placa de aluminio según la figura 6 en los lados laterales de la silla de ruedas convencional, de tal forma que funcione como soporte del conjunto de ruedas dispuesto en estrella y optimice la repartición de peso como se demuestra mediante cálculos matemáticos.

La estabilidad se consigue mediante tres puntos de apoyo y sujeción de la placa con el chasís de la estructura de la silla de ruedas, como se observa nuevamente en la figura 6 .

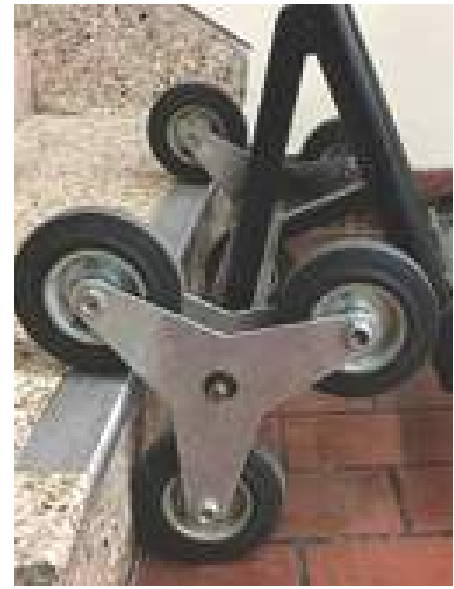

Figura 5. Pruebas del prototipo del sistema mecánico Elaboración propia

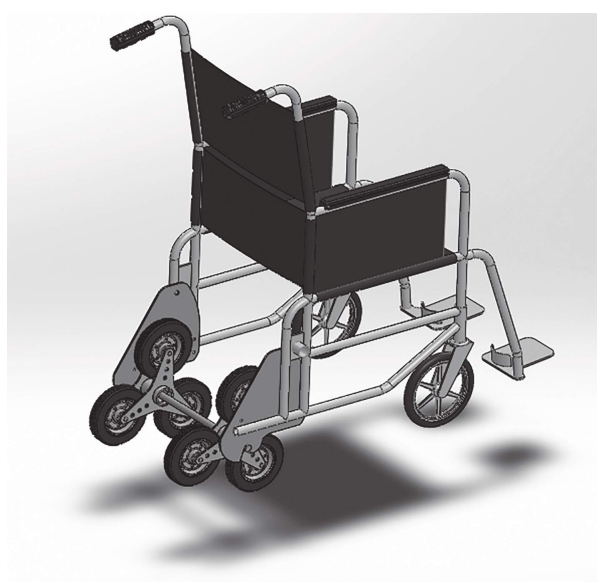

Figura 6: Silla de ruedas convencional, con las ruedas traseras desensambladas Elaboración propia

\subsection{Sistema eléctrico}

Para lograr el desplazamiento motriz de la silla, se han previsto dos motores eléctricos de corriente continua con reductor de velocidad de 10 a 1 y eje de salida perpendicular a cada motor. Se monta un motor eléctrico en cada rueda de tracción, que va sobre cada placa metálica 
del sistema mecánico diseñado. Cada motor tiene una potencia de 450 watts, 17,5 amperios, 24 voltios DC y $2000 \mathrm{rpm}$.

Asimismo, se han instalado dos baterías de 12 voltios de plomo-ácido sulfúrico conectadas en serie como fuente de energía eléctrica para alimentar los motores eléctricos. Cada batería entrega una corriente máxima de 518 amperios, y tiene una capacidad de carga de 75 amperios por hora. Cada batería a máximo consumo dura aproximadamente 4 horas.

El driver de potencia cuenta con una capacidad de 50 amperios. Esto se debe a que, cuando la silla se encuentra funcionando, es posible que la persona discapacitada pueda chocar contra una pared o una puerta; entonces, la corriente de trabajo se eleva de forma drástica. Este sistema de locomoción cuenta con sensores que permiten controlar de manera adecuada la velocidad en una pendiente, tanto de bajada como de subida.

El FPGA myRIO cuenta con un software que asiste al sistema en todo momento de su traslación.

En la figura 7 se presenta el mecanismo ya montado, con los motores eléctricos y el acoplamiento.

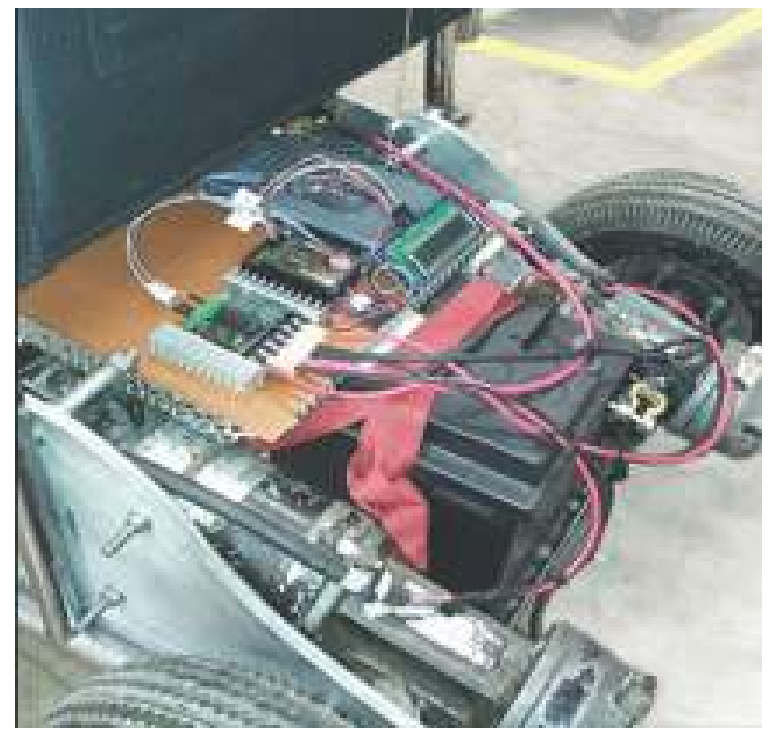

Figura 7. Mecanismo electrónico de la silla de ruedas Elaboración propia 


\subsection{Sistema electrónico y de control}

El sistema de control automatizado está basado en un software de desarrollo de sistemas, conocido como LabVIEW, con las herramientas propias del procesador myRIO de National Instruments. El FPGA (Field-Programmable Gate Array) es un dispositivo programable que permite emular cualquier circuito integrado para traducir circuitos que ya no se fabrican en la actualidad. La arquitectura de myRIO está basada en un FPGA.

El sensor Myo se encarga de mandar la señal al controlador mediante los pulsos musculares del usuario. Este sensor es utilizado como un brazalete en el antebrazo y, mediante la detección de ciertos patrones de movimientos musculares, envía distintas señales encargadas de la locomoción del sistema, ya sea hacia adelante, voltear a la derecha, izquierda o en reversa.

La programación se desarrolló de tal manera que el sensor grabara en su memoria determinados patrones de movimiento muscular y los tradujera en señales que el controlador interpreta como distintos tipos de movimiento de la silla. Todo esto se logró gracias al programa en LabView.

El sensor que permite la locomoción automática de la silla de ruedas es el sensor Myo, el cual permite la interpretación de un patrón muscular para que el controlador mande la señal de movimiento.

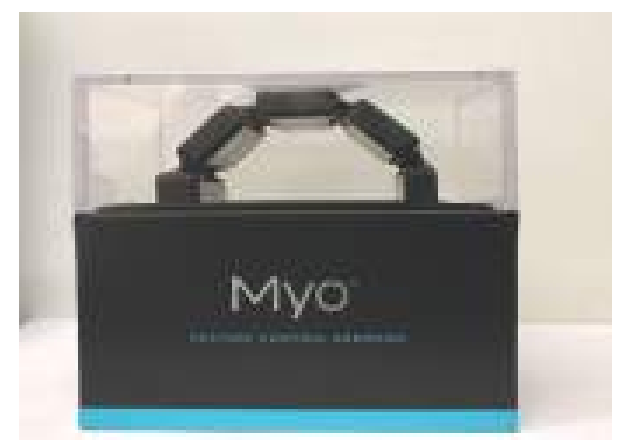

Figura 8. Sensor Myo Elaboración propia

La siguiente serie de figuras indica el funcionamiento del sistema de control del sistema de locomoción: 


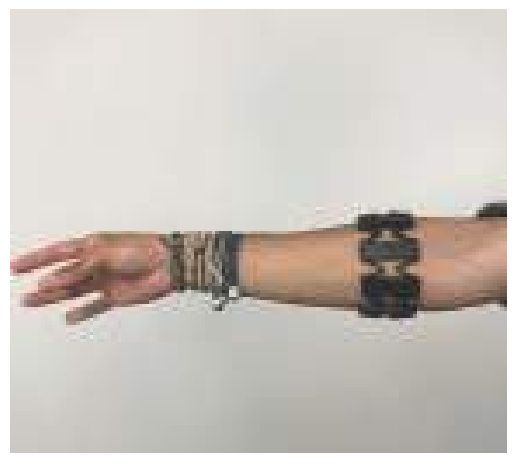

Figura 9. Patrón de movimiento de activación del sensor de pulsos Elaboración propia

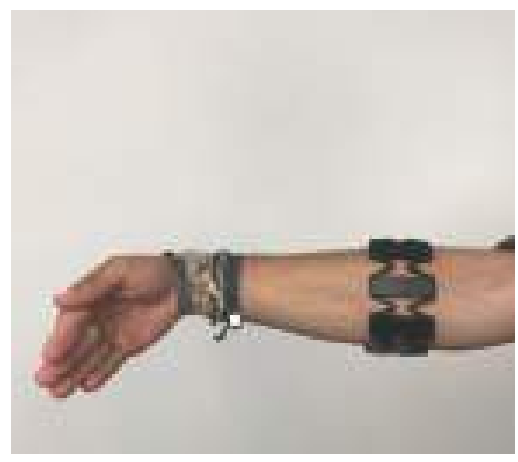

Figura 11. Patrón de movimiento de giro a la izquierda Elaboración propia

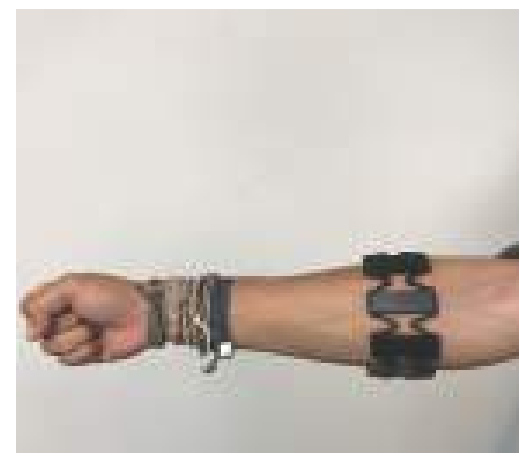

Figura 10. Patrón de movimiento de desplazamiento lineal

Elaboración propia

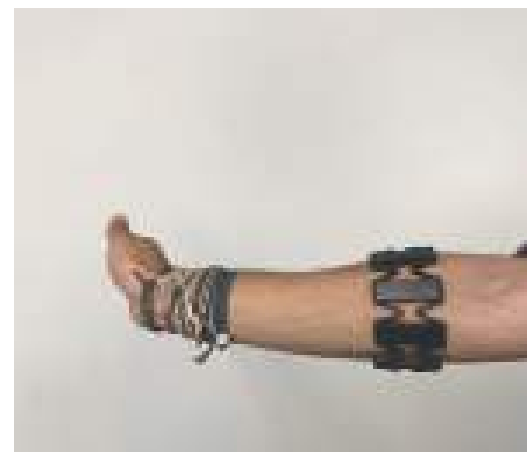

Figura 12. Patrón de movimiento de giro a la derecha

Elaboración propia

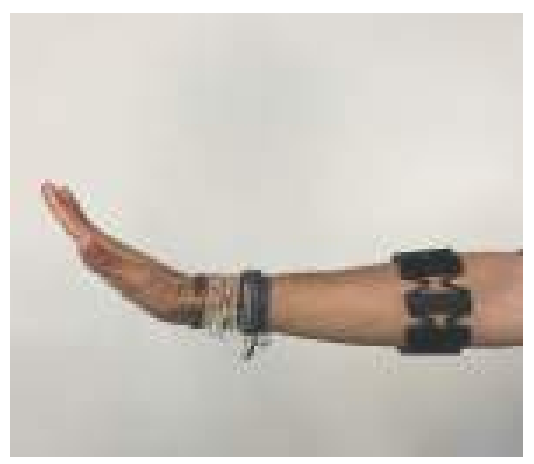

Figura 13. Patrón de movimiento de reversa

Elaboración propia 
Actualmente se cuenta con sensores que detectan el consumo de corriente de los motores eléctricos, hasta un máximo de 25 amperios, con corrientes pico de hasta 50 amperios en el mando de potencia. Este driver o mando de potencia recibe el tren de pulsos PWM (Pulse-Width Modulation) del controlador myRIO.

La comunicación entre el sensor Myo y la computadora a bordo de la silla es por señal inalámbrica Bluetooth; el computador se comunica a través de un cable USB con la plataforma Arduino. Este controlador tiene conexión digital con la plataforma de control MyRIO donde se ejecuta el programa principal de control, que da lugar a las señales PWM, de control de los motores DC que mueven la silla de ruedas.

El diagrama de la figura 14 detalla el flujo de comunicación de datos entre el sensor, el controlador y los periféricos, así como el tipo de señal que se envía y recibe y el orden en que estas señales son decodificadas hasta llegar a controlar el sistema integral y ofrecer el sistema de adquisición de datos.

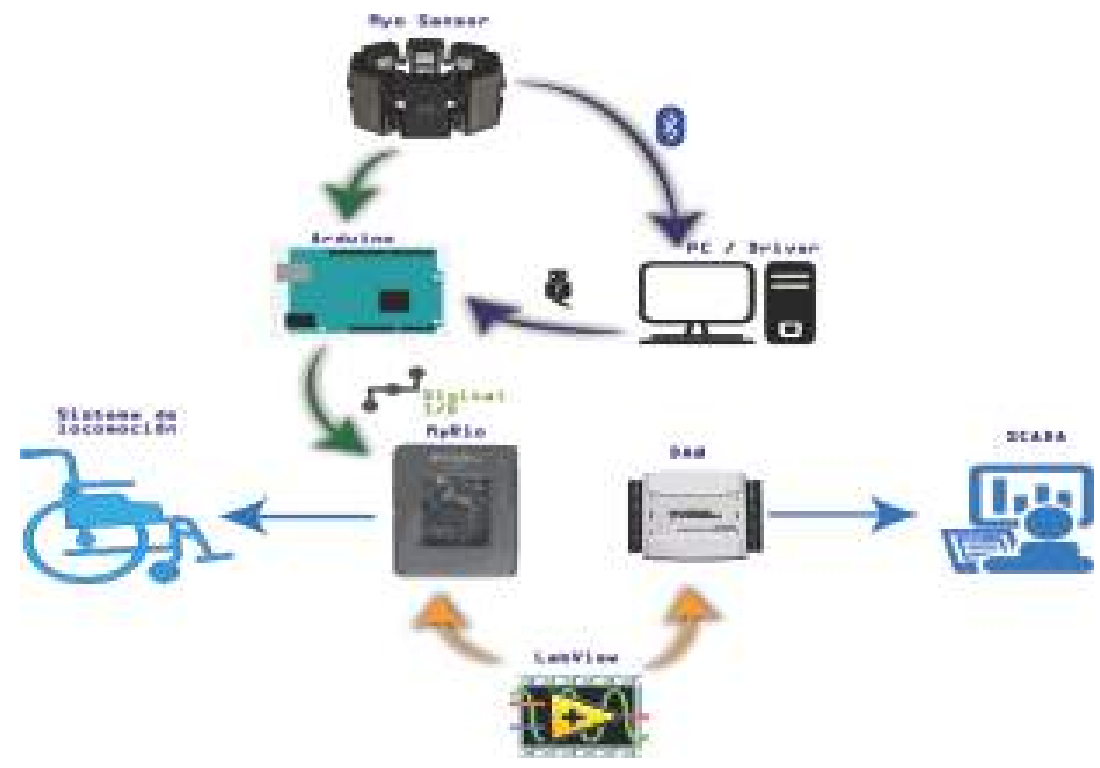

Figura 14. Patrón de movimiento de giro a la derecha Elaboración propia 
El computador a bordo ejecuta una rutina en el software de desarrollo gráfico LabView para generar el programa de supervisión, control y adquisición de datos Scada. Los sensores de corriente miden el amperaje de consumo de cada motor de las ruedas.

Además, el sistema posee dos sensores de voltaje para cada una de las dos baterías y un sensor para el voltaje total, pues no necesariamente funcionan las dos baterías al mismo voltaje.

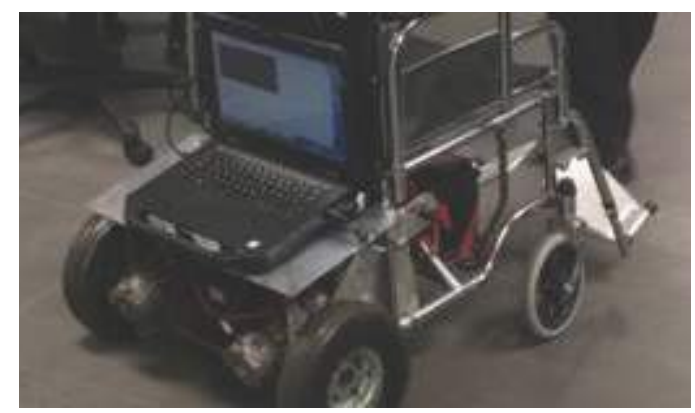

Figura 15. Hardware de control acoplado a la silla de ruedas

Elaboración propia

El sistema Scada almacena la data histórica de los sensores en una hoja de cálculo.

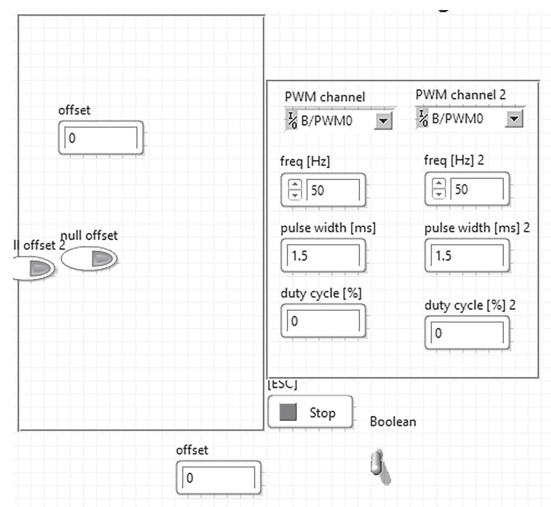

motor izquierdo: canal1

motor derecho: canal2

Figura 16. Control de un servomotor por medio de PWM con FPGA myRIO

Elaboración propia 


\subsection{Programación}

La facilidad de conexión y de comunicación con el software LabVIEW y el procesador myRIO permite reducir las horas de programación, con lo cual logra obtener un código más limpio, más sencillo de comprender y de resultados óptimos.

El algoritmo de control se desarrolla inicialmente en un sistema de lazo abierto que, con los encoder, se convertirá en un lazo de control cerrado. A continuación, se presenta el algoritmo de control.

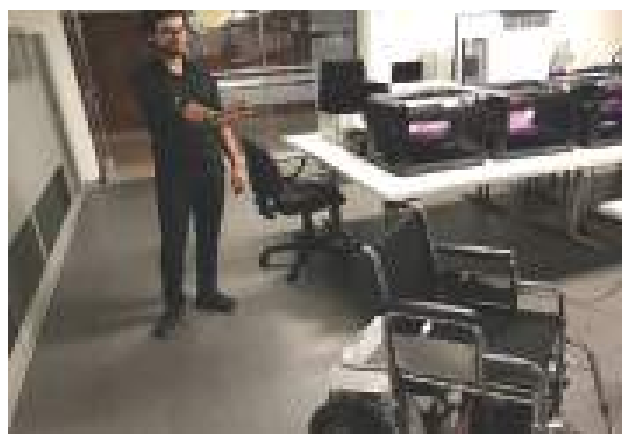

Figura 17. El patrón de movimiento con el antebrazo permite a la silla de ruedas moverse en reversa Elaboración propia

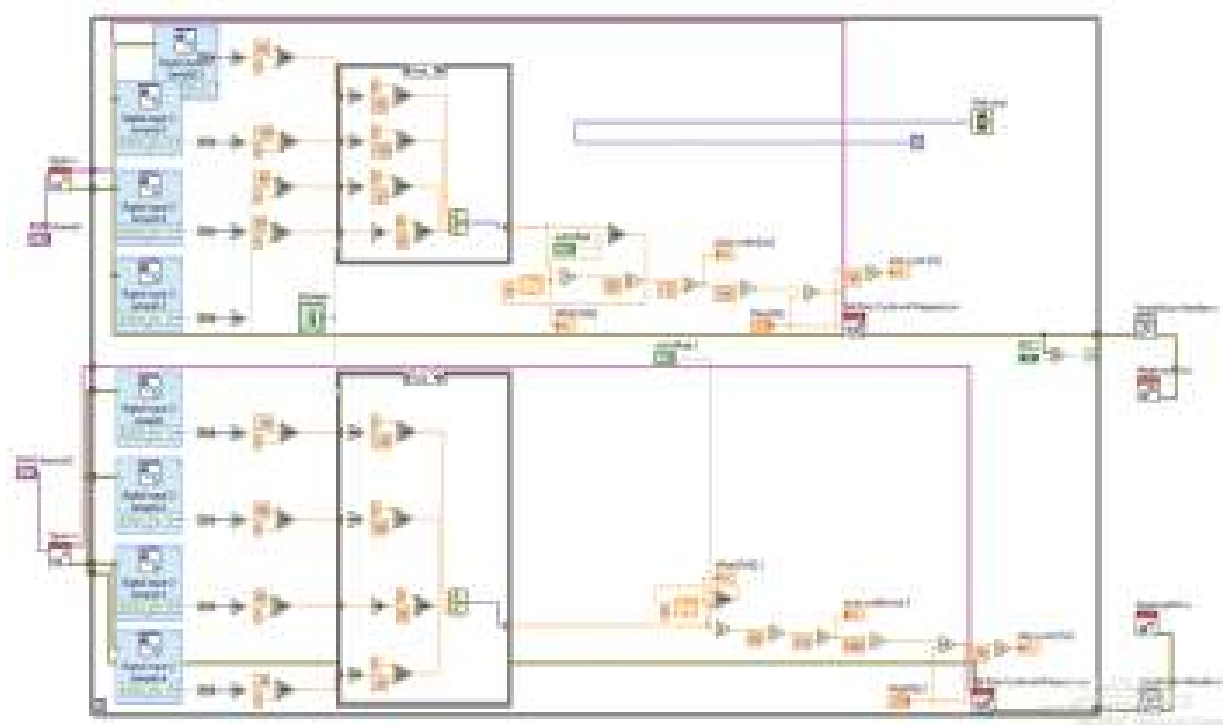

Figura 18. Algoritmo de control Elaboración propia 
El control consiste en determinar un patrón de movimientos musculares con el antebrazo. El controlador detectará los pulsos musculares y los asociará con un tipo de movimiento (adelante, reversa, izquierda, derecha) y enviará la señal a los motores, los cuales realizarán los movimientos correspondientes.

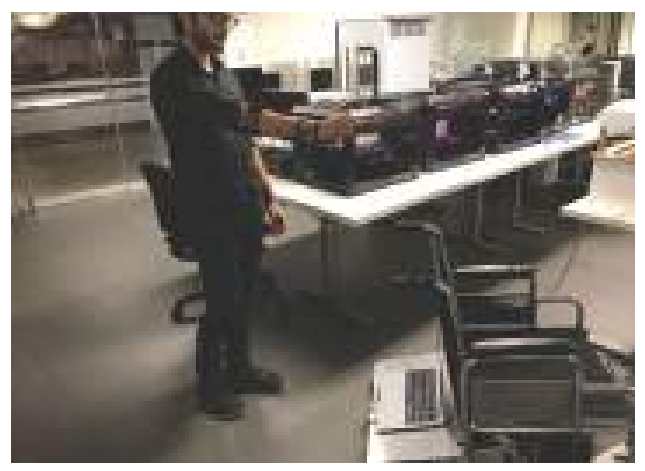

Figura 19. Asimismo, otro patrón de movimiento permite a la silla de ruedas ir en línea recta Elaboración propia

\section{CONCLUSIONES}

\subsection{Logros alcanzados en el proyecto}

El sistema mecánico diseñado es de fácil montaje y desmontaje para una silla de ruedas convencional.

El software programado myRIO nos permite actualmente desplazar la silla convencional en sus cuatro direcciones: adelante, atrás, izquierda, y derecha. Además, puede superar pendientes de hasta diez grados de inclinación.

El uso de las herramientas tecnológicas LabVIEW / myRIO nos permite resolver problemas serios y de importancia social, como el tema sensible de la discapacidad en el país. 


\section{REFERENCIAS}

Doering, E. (2014). National Instruments myRIO: Project Essentials Guide. Texas: National Technology and Science Press.

Goñi, J. C. (2011). Máquinas, instrumentos y procesos de manufactura. Lima: Universidad de Lima, Fondo Editorial.

Instituto Nacional de Estadística e Informática (2015). Perú: Características de la población con discapacidad. Lima: INEI.

Torres Román, M. J. (2012). Diseño de adaptación y elaboración de un manual de procedimientos para transformar sillas de ruedas convencionales en sillas para pacientes con problemas neurológicos y escasos recursos económicos. Lima: Pontificia Universidad Católica del Perú. 